\title{
Ordering of metallic quantum dots
}

\author{
David Salac and Wei Lü \\ Department of Mechanical Engineering, University of Michigan, Ann Arbor, Michigan 48109
}

(Received 11 April 2006; accepted 27 June 2006; published online 15 August 2006)

\begin{abstract}
This letter proposes a mechanism for the ordering of metallic quantum dots without coherent lattice or lattice mismatch with the substrate so that elasticity may not account for the phenomena. The authors show that contact potential induces repulsive charge clouds in the substrate. The size-dependent repulsion and van der Waals attraction lead to ordered nanoscale structures. (C) 2006 American Institute of Physics. [DOI: 10.1063/1.2337006]
\end{abstract}

Recent experiments show that ordered metallic quantum dots may form spontaneously on a semiconductor substrate, such as $\mathrm{Cu}$ on $\mathrm{TiO}_{2},{ }^{1} \mathrm{Au}$ on $\mathrm{Si}(111),{ }^{2} \mathrm{Fe}$ on $\mathrm{NaCl}(100),{ }^{3}$ and $\mathrm{CoSi}_{2}$ on $\mathrm{Si}(111) .{ }^{4}$ Despite their large collective surface area, the densely packed dots did not coalesce, but maintained small distances from one another. The observations call for a repulsive force when they are close. In a traditional quantum dot system, such as Ge dots on a Si substrate, the repulsion is achieved by elastic interaction. This letter proposes a mechanism for metallic dots. These systems may not involve coherent lattice or lattice mismatch so that elasticity cannot explain the phenomena. A qualitative understanding is provided in the following. The metallic dots and the substrate have different Fermi levels. When they are brought in contact, charge transfer occurs. Take $n$-doped semiconductor as an example. As a result of the charge transfer, negative charges accumulate at the metal interface while a cloud of positive charges form in the substrate. This configuration is known as electric double layer. Thus, underneath a metallic dot, there is a charge cloud in the semiconductor. The exact shape and density of this cloud depend on factors such as the strength of contact potential and contact geometry. When the dot moves, the charge cloud moves with it. When two dots approach each other, the accompanying charge clouds overlap, leading to a repulsive force that prevents them to coalesce. This possibility is exciting since the repulsive force may help us to assemble a lattice of dots such as colloidal crystals.

Electric double layers form the basis of $p$ - $n$ junctions and metal-semiconductor contacts. ${ }^{5}$ However, existing work focuses on electronic properties. Little work has been done to investigate the role of electric double layers on selfassembly. Several differences distinguish the system of metallic dots from colloidal particles in a liquid electrolyte. First, in a semiconductor, say $n$ doped, electrons are mobile, but positive ions do not. By contrast, in a liquid electrolyte, ions of both signs are mobile. Second, charge distribution leads to different degrees of screening in the two systems. Imagine two colloidal particles carrying negative charges. Each of these particles will be surrounded by a layer of positive charges in the solution, which significantly reduces the repulsion between them. The screening effect in metallic dots is much weaker since they are only partially surrounded by opposite charges in the substrate. Third and most importantly, colloidal particles usually carry fixed charges while

\footnotetext{
a) Author to whom correspondence should be addressed; electronic mail: weilu@umich.edu
}

metallic dots keep constant contact potential, which leads to a different energy landscape. We find that when the contact is smaller than a characteristic length, the size of the charge cloud is no longer determined by the doping level or the free carrier concentration, but instead by the size and shape of the interface, which strongly affects the interaction among dots. The electric double layers form an energy barrier that keeps two dots separated. If the barrier is passed, van der Waals attraction will bring the dots all the way together. Surface energy will then drive them to combine into a large dot.

Consider metallic dots on a semi-infinite substrate. A coordinate is attached so that the substrate occupies the half space below the $x_{1}-x_{2}$ plane $\left(x_{3}<0\right)$. Inside the semiconductor, the electric potential $\phi$ and the charge density $\rho$ vary with the position. The electrostatic field obeys the Poisson equation, $-\varepsilon_{s} \nabla^{2} \phi=\rho$, where $\varepsilon_{s}$ is the permittivity of the substrate. The charge density $\rho$ is a function of the electron density, hole density, and donor and acceptor densities. The electrons obey the Fermi-Dirac statistics. The general form of the function, $\rho$, can be rather cumbersome. For an $n$-doped nondegenerate semiconductor with fully ionized donors, which is typical in many situations, the charge density is given by $\rho=q N_{d}\left(1-\exp \left(q \phi k_{B} T\right)\right){ }^{5}$ Here $N_{d}$ is the donor density in the substrate, $q$ the charge of one electron, $k_{B}$ Boltzmann's constant, and $T$ the temperature. A characteristic length scale known as the Debye length is defined by $l_{D}=\sqrt{\varepsilon_{s} k_{B} T\left(q^{2} N_{d}\right)}$. A characteristic voltage is defined by $\phi_{0}=k_{B} T q$. Denote the normalized potential by $\psi=\phi \phi_{0}$ and normalize the length by $l_{D}$, we have

$$
-\nabla^{2} \psi=1-e^{\psi}
$$

This equation describes the potential distribution in the substrate.

Unlike colloidal particles holding constant charges, ${ }^{6}$ the metallic dots hold constant contact potential $\phi_{s}$ when they move on the substrate. The Gibbs free energy is given by $G_{e}=-\int_{0}^{\phi_{s}} Q_{s}\left(\phi_{s}^{\prime}\right) d \phi_{s}^{\prime}$, where $Q_{s}$ is the charge accumulated on the interface. ${ }^{7}$ However, direct application of this expression requires solving Eq. (1) for a series of surface potentials to construct the $Q_{s}\left(\phi_{s}^{\prime}\right)$ curve, which is computationally intensive. Our analysis showed that the free energy can be expressed by a volume integration in the current electrostatic state without the need to calculate the history of $Q_{s}\left(\phi_{s}^{\prime}\right)$, which gives $G_{e}=\int_{V}\left[\int_{0}^{\phi} \rho\left(\phi^{\prime}\right) d \phi^{\prime}-\varepsilon_{s}|\nabla \phi|^{2} 2\right] d V$. The integration extends over the volume of the substrate and air. The air region has $\rho=0$, where the free energy reduces to the ordi- 


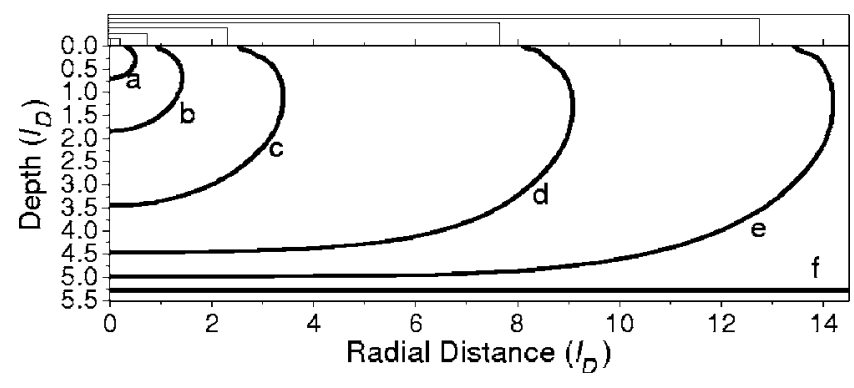

FIG. 1. Contour of $4.5 \% \psi_{s}$ for disks with radii (a) $0.232 l_{D}(30 \mathrm{~nm})$, (b) $0.773 l_{D}(100 \mathrm{~nm}),(\mathrm{c}) 2.32 l_{D}(300 \mathrm{~nm}),(\mathrm{d}) 7.73 l_{D}(1 \mu \mathrm{m})$, and (e) $12.36 l_{D}$ $(1.6 \mu \mathrm{m})$ and (f) infinite contact. The size of the charge cloud in the semiconductor becomes highly dependent on the dimension of the contact interface when the contact falls into nanoscale.

nary form of $-\int_{V} \varepsilon_{a}|\nabla \phi|^{2} d V 2$ with $\varepsilon_{a}$ being the permittivity of air. Calculation of $\nabla \phi$ can be avoided by applying the divergence theorem, which gives

$$
\begin{aligned}
\frac{G_{e}}{N_{d} k_{B} T l_{D}^{3}}= & \int_{V_{\text {sub }}}\left[1+\frac{\psi}{2}+\left(\frac{\psi}{2}-1\right) \exp (\psi)\right] d V \\
& +\frac{1}{2} \int_{A} \psi\left[\frac{\varepsilon_{a}}{\varepsilon_{s}}\left(\frac{\partial \psi}{\partial x_{3}}\right)_{x_{3}=0^{+}}-\left(\frac{\partial \psi}{\partial x_{3}}\right)_{x_{3}=0^{-}}\right] d A .
\end{aligned}
$$

Here the volume integration extends only in the substrate $V_{\text {sub }}$ and the area integration extends on the contact interface. The terms $\left(\partial \psi \partial x_{3}\right)_{x_{3}=0^{+}}$and $\left(\partial \psi \partial x_{3}\right)_{x_{3}=0^{-}}$are the potential gradients at the contact interface calculated from the air and the substrate region, respectively. In fact, the integrand of the area integration vanishes in the noncontact substrate surface due to the continuality of the electric displacement.

An analytical solution to Eq. (1) cannot be obtained due to the nonlinear charge density. We developed a spectral and interface relaxation method for both high accuracy and computational speed. The electric potential satisfies the Laplace equation in the air and Eq. (1) in the substrate. The two fields have prescribed contact potential at the contact interface, and continuous normal electric displacement across the noncontact area. We temporally relax the second requirement by assuming a potential distribution $\psi_{b}\left(x_{1}, x_{2}\right)$ on the entire substrate surface. This function satisfies the contact potential at the contact interface and has arbitrary values in the noncontact area. Then $\psi_{b}\left(x_{1}, x_{2}\right)$ serves as a given potential boundary condition so that the electric fields in the air and the substrate can be solved independently. In the substrate $\left(x_{3}\right.$ $<0$ ) we apply two-dimensional Fourier transform within the $x_{1}-x_{2}$ plane to convert the three-dimensional partial differential equation into a set of ordinary differential equations in the $x_{3}$ direction. These tridiagonal systems of equations can be solved efficiently. The field in the air $\left(x_{3}>0\right)$ can be solved analytically in Fourier space. Generally the two half-spaces solved separately will not satisfy the continuity of electric displacement. The discontinuity corresponds to a surface charge density of $\sigma=\left(\partial \psi \partial x_{3}\right)_{x_{3}=0^{-}}-\left(\varepsilon_{a} \varepsilon_{s}\right)$ $\times\left(\partial \psi \partial x_{3}\right)_{x_{3}=0^{+}}$, which increases the energy by $G_{b}=\int_{A_{\text {noncontact }}} \sigma \psi_{b} d A 2$. Minimizing the functional with the Langevin steepest decent approach gives $\beta \partial \psi_{b} \partial t=-\delta G_{b} \delta \psi_{b}$, namely,

$$
\beta \frac{\partial \psi_{b}}{\partial t}=\frac{\varepsilon_{a}}{\varepsilon_{s}}\left(\frac{\partial \psi}{\partial x_{3}}\right)_{x_{3}=0^{+}}-\left(\frac{\partial \psi}{\partial x_{3}}\right)_{x_{3}=0^{-}},
$$

where $\beta$ is a parameter to control the rate of convergence. The evolution of $\psi_{b}\left(x_{1}, x_{2}\right)$ reduces and eventually eliminates the discontinuity when it reaches the actual potential distribution.

The system of $\mathrm{CoSi}_{2}$ disks on $n$-doped silicon substrate was taken as an example in our study. The silicon substrate has $\varepsilon_{s}=11.7 \varepsilon_{0}$, where $\varepsilon_{0}$ is the permittivity of vacuum. Using $\varepsilon_{0}=8.85 \times 10^{-12} \mathrm{Fm}, \quad q=1.60 \times 10^{-19} \mathrm{C}, \quad k_{B}=1.38$ $\times 10^{-23} \mathrm{JK}, T=300 \mathrm{~K}$, and a typical donor density of $N_{d}=10^{15} \mathrm{~cm}^{-3}$, we have $l_{D}=129.4 \mathrm{~nm}$ and $\phi_{0}=2.59$ $\times 10^{-2} \mathrm{~V}$. We prescribed the normalized contact potential $\psi_{s}=\phi_{s} \phi_{0}$ at the metal-semiconductor interface and zero potential deep inside the substrate. Contact potential $\phi_{s}$ can be measured experimentally or related to other quantities by $\phi_{s}=\phi_{B}-V_{n}$, where $\phi_{B}$ is the barrier height between the metal and semiconductor and $q V_{n}=E_{C}-E_{F}$ is the energy difference between the conduction band $E_{c}$ and the Fermi level $E_{F}$ in the semiconductor. For $\mathrm{CoSi}_{2}$ on $\mathrm{Si}(111)$ the barrier height is $\phi_{B}=0.67 \mathrm{eV}{ }^{8}$ The silicon substrate has $E_{C}-E_{F}=1.1 \mathrm{eV} .^{5}$ Then the contact potential is $\phi_{s}=-0.4 \mathrm{~V}$ or $\psi_{s}=-15.4$. We calculated van der Waals interaction by pairwise summation of intermolecular forces, ${ }^{9}$ namely, $G_{\mathrm{vdW}}=\sum_{i} \Sigma_{i>j}\left(-B r_{i j}^{6}\right)$. Here $B$ is the London constant and $i$ and $j$ count over all atoms or molecules. The term $r_{i j}$ is the distance between two atoms. The London constant relates to the Hamaker constant $A$ by $A=\pi^{2} \rho_{1} \rho_{2} B$, where $\rho_{1}$ and $\rho_{2}$ are the atomic density of two interacting bodies. The Hamaker constant can be determined from the index of refraction. For $\mathrm{CoSi}_{2}$, the index value of

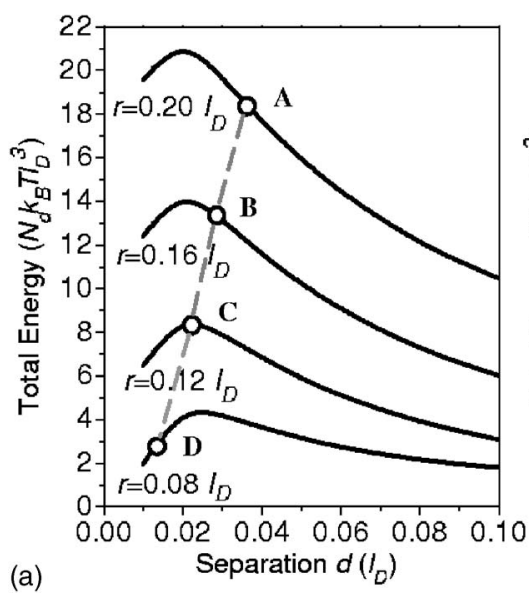

(a)

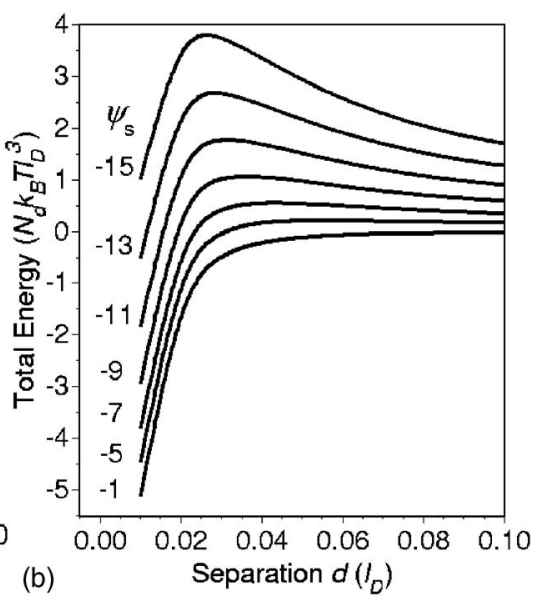

FIG. 2. Electrostatic and van der Waals energies for disks with (a) various radii and (b) with radius of $0.08 l_{D}$ for various contact potentials. 
1.3 gives a Hamaker constant of $1.8 \times 10^{-19} \mathrm{~J}$ or a London constant of $2.0 \times 10^{-77} \mathrm{~J} \mathrm{~m}^{6}{ }^{6}{ }^{10}$

Computations suggest that the size of the charge cloud in the semiconductor becomes highly dependent on the dimension of the contact interface when the contact falls into nanoscale. This trend is shown in Fig. 1, which gives the potential contour of $4.5 \% \psi_{s}$ for various disk radii. This is also the charge density contour of half of the maximum charge density at the contact interface since $\rho=q N_{d}\left(1-e^{\psi}\right)$. The enclosure of the contour can be viewed as the size of the charge cloud since the charge density decays sharply outside. The curve for infinite contact was obtained from a onedimensional model. The depth of the charge cloud increases with the contact size and quickly approaches that of an infinite contact, i.e., curve $f$. Thus a large contact causes the charge cloud to expand most laterally, with a little increase in the depth. Figure 2(a) shows the total electrostatic and van der Waals energies for two disks with various radius $r$ and disk separation distance $d$. The zero energy state is defined when two disks are infinitely separated. An energy barrier appears at a separation of approximately $0.02 l_{D}$, below which van der Waals interaction dominates and drives two disks to contact. The barrier depends on the disk size and the contact potential. Larger disks have higher barrier and are less likely to combine. The percentage of the substrate surface covered by metal is a linear function of $r d$. For instance, a triangular lattice of disks with $r d=5.3$ correspond to a coverage of 0.76 . For a given coverage, the energy barrier determines the disk size. The dashed curve, which is the $r d=5.3$ contour, illustrates the idea. This curve passes point $C$, the maximum of the $r=0.12 l_{D}$ curve. Smaller disks, such as point $D$, tend to grow since there is no barrier for them to coalesce. On the other hand, larger disks such as point $A$ or $B$ are energetically unfavorable. These effects lead to disk ra- dius and separation defined by point $C$. Higher temperature leads to larger Debye length and thus larger physical disk size and separation. This prediction is consistent with experiments, which showed that increasing the annealing temperature in the $\mathrm{TiSi}_{2} \mathrm{Si}(001)$ system produced disks with larger size and spacing. ${ }^{11}$ Figure 2(b) shows the barrier for two small disks of radius $0.08 l_{D}$ at different contact potentials. When the magnitude of the contact potential is small, the repulsion cannot overcome van der Waals attraction and there is no barrier for coalescence. Feature size may be engineered by applying a bias voltage to the substrate to tune the energy barrier. Designed voltage pattern has been shown to direct the self-assembly of adsorbate molecules. ${ }^{12}$ Similar concept may also be applied to achieve various configurations of metallic quantum dots.

The authors acknowledge financial support from National Science Foundation Career Award No. DMI-0348375.

${ }^{1}$ D. L. Carroll, M. Wagner, M. Ruhle, and D. A. Bonnell, Phys. Rev. B 55, 9792 (1997).

${ }^{2}$ M. Hugelmann and W. Schindler, Appl. Phys. Lett. 85, 3608 (2004).

${ }^{3}$ Z. Gai, B. Wu, J. P. Pierce, G. A. Farnan, D. J. Shu, M. Wang, Z. Y. Zhang, and J. Shen, Phys. Rev. Lett. 89, 235502 (2002).

${ }^{4}$ F. M. Ross, P. A. Bennett, R. M. Tromp, J. Tersoff, and M. Reuter, Micron 30, 21 (1999).

${ }^{5}$ S. M. Sze, Physics of Semiconductor Devices (Wiley, New York, 1981).

${ }^{6}$ K. A. Sharp and B. Honig, J. Phys. Chem. 94, 7684 (1990).

${ }^{7}$ L. Landau and E. Lifshitz, Electrodynamics of Continuous Media (Pergamon, Oxford, 1984).

${ }^{8}$ R. T. Tung, Mater. Chem. Phys. 32, 107 (1992).

${ }^{9}$ W. R. Bowen and F. Jenner, Adv. Colloid Interface Sci. 56, 201 (1995).

${ }^{10}$ R. H. French, J. Am. Ceram. Soc. 83, 2117 (2000).

${ }^{11}$ W. Yang, F. J. Jedema, H. Ade, and R. J. Nemanich, Thin Solid Films 308, 627 (1997).

${ }^{12}$ W. Lu and D. Salac, Phys. Rev. Lett. 94, 146103 (2005). 